\title{
Pooled Analysis of Clinical Outcome of Patients with Chemorefractory Metastatic Colorectal Cancer Treated within Phase I/II Clinical Studies Based on Individual Biomarkers of Susceptibility: A Single-Institution Experience
}

\author{
Andrea Sartore-Bianchi ${ }^{1}$ - Alessio Amatu ${ }^{1} \cdot$ Erica Bonazzina $^{1} \cdot$ Stefano Stabile $^{1}$. \\ Laura Giannetta $^{1}$ - Giulio Cerea ${ }^{1}$. Ilaria Schiavetto ${ }^{1} \cdot$ Katia Bencardino $^{1}$. \\ Chiara Funaioli $^{1} \cdot$ Riccardo Ricotta $^{1}$ - Tiziana Cipani ${ }^{1} \cdot$ Michele Schirru $^{1}$. \\ Valentina Gambi ${ }^{1}$. Laura Palmeri ${ }^{1}$. Giulia Carlo-Stella ${ }^{1}$. Francesca Rusconi ${ }^{1}$. \\ Sara Di Bella ${ }^{1}$ - Giovanni Burrafato ${ }^{1}$ - Andrea Cassingena ${ }^{1} \cdot$ Emanuele Valtorta $^{1}$. \\ Calogero Lauricella ${ }^{1}$ - Federica Pazzi ${ }^{1}$ - Alessandra Gambaro ${ }^{1}$ - Silvia Ghezzi ${ }^{1}$ • \\ Giovanna Marrapese $^{1} \cdot$ Emiliana Tarenzi $^{1} \cdot$ Silvio Veronese $^{1} \cdot$ Mauro Truini $^{1}$. \\ Angelo Vanzulli ${ }^{1,2} \cdot$ Salvatore Siena ${ }^{1,2}$
}

Published online: 1 July 2017

(C) The Author(s) 2017. This article is an open access publication

\begin{abstract}
Background Patients with metastatic colorectal cancer (mCRC) refractory to standard therapies have a poor prognosis. In this setting, recruitment into clinical trials is warranted, and studies driven by selection according to individual tumor molecular characteristics are expected to provide added value. Objective We retrospectively analyzed data from patients with $\mathrm{mCRC}$ refractory to or following failure of standard therapies who were enrolled into phase I/II clinical studies at the Niguarda Cancer Center based on the presence of a specific molecular profile expected to represent the target of susceptibility to the experimental drug(s).

Patients and Methods From June 2011 to May 2016, 2044 patients with mCRC underwent molecular screening. Eighty patients $(3.9 \%)$ were enrolled in ad hoc studies; the median age was 60 years (range 36-86) and the median number of previous treatment lines was five (range 2-8). Molecular char-
\end{abstract}

Salvatore Siena

salvatore.siena@ospedaleniguarda.it

1 Niguarda Cancer Center, Grande Ospedale Metropolitano Niguarda, Piazza Ospedale Maggiore, 3, 20162 Milan, Italy

2 Dipartimento di Oncologia e Emato-Oncologia, Università degli Studi di Milano, Milan, Italy acteristics exploited within these studies were $M G M T$ promoter hypermethylation (48.7\%), HER2 amplification (28.8\%), $B R A F^{V 600 E}$ mutation (20\%), and novel gene fusions involving $A L K$ or NTRK (2.5\%).

Results One patient (1\%) had RECIST (Response Evaluation Criteria In Solid Tumors) complete response (CR), 13 patients (16.5\%) experienced a partial response (PR), and 28 (35\%) stable disease (SD). Median progression-free survival (PFS) was 2.8 months (range $2.63-3.83$ ), with $24 \%$ of patients displaying PFS $>5$ months. Median growth modulation index (GMI) was 0.85 (range $0-15.61$ ) and $32.5 \%$ of patients had GMI $>1.33$. KRAS exon 2 mutations were found in $38.5 \%$ of patients, and among the 78 patients with known $K R A S$ status, those with wildtype tumors had longer PFS than those with mutated tumors (3.80 [95\% CI 2.80-5.03] vs. 2.13 months [95\% CI $1.77-$ 2.87], respectively, $p=0.001$ ). Median overall survival (OS) was 7.83 months (range 7.17-9.33) for all patients, and patients with $K R A S$ wild-type tumors had longer OS than those with mutated tumors (7.83 [95\% CI 7.33-10.80] vs. 7.18 months [95\% CI 5.63-9.33], respectively, $p=0.06$ ).

Conclusions This single-institution retrospective study indicates that in a heavily pretreated population approximately $4 \%$ of mCRC tumors display a potential actionable molecular context suitable for therapeutic intervention. Application of molecular selection is challenging but improves clinical outcome even in later lines of treatment. 


\section{Key Points}

Treatment of metastatic colorectal cancer (mCRC) patients driven by selection according to individual tumor molecular characteristics is expected to provide enhanced clinical benefit.

In this single-institution retrospective analysis, 3.9\% of 2044 patients were found to harbor biomarkers for $\mathrm{ad} \mathrm{hoc}$ phase I-II clinical studies, including $M G M T$ promoter hypermethylation, HER2 amplification, $B R A F^{V 600 E}$ mutation, and gene fusions involving $A L K$ or NTRK.

14 patients had an objective response, and 28 stable disease. Median progression-free survival (PFS) was 2.8 months, with $24 \%$ of patients displaying PFS $>5$ months.

\section{Introduction}

Colorectal cancer (CRC) remains a significant cause of morbidity and mortality worldwide and many patients present with metastatic disease despite large-scale screening programs in various countries [1]. With the only exception of patients presenting with oligometastatic lesions confined to the liver or lung amenable to resection $[2,3]$, metastatic disease is considered incurable. In cases where treatment with curative intent is excluded, patients are typically given a combination of cytotoxic chemotherapy, often in conjunction with a targeted therapy.

Although advances in systemic therapy have been made, the 5-year survival rate in this setting is still $12.5 \%$ [4], with acquired resistance to therapy being the main reason for treatment failure [5]. Indeed, resistance to targeted therapy, as displayed by disease progression, is observed after a median of 4 months on epidermal growth factor receptor (EGFR) antagonists [45]. The discovery of RAS mutations in CRC as a mechanism of innate resistance to these therapies has been an important advance and has ameliorated their clinical use. However, there is an unmet need for effective therapeutic strategies after secondary resistance.

We have previously demonstrated that different molecular alterations that drive resistance can occur simultaneously in the same patient [7]. Identifying relevant molecular subtypes within this heterogeneous disease and matching patients with appropriate single agents or combinations of targeted therapies at resistance is crucial to therapeutic progress [8]. Therefore, recruitment into precision oncology clinical trials based on selection according to individual tumor molecular characteristics is expected to provide added value.

We retrospectively collected data from patients with metastatic CRC (mCRC) resistant to standard therapies treated at the Niguarda Cancer Center (NCC) (Milan, Italy) in phase I/II clinical studies based on the presence of specific tumor molecular profiles conferring susceptibility to experimental drugs, and performed a pooled analysis for measuring results according to main clinical and other molecular variables.

\section{Methods}

\subsection{Patients}

We retrospectively collected data from patients with mCRC resistant to standard therapies treated at $\mathrm{NCC}$ between June 2011 and May 2016 in phase I/II clinical studies, including one phase I first-in-human study, based on the presence of specific biomarkers that confer susceptibility to experimental drugs (Table 1). These included tumor genetic alterations (i.e., gene mutations, amplifications, or fusions) or a certain genetic context (i.e., methylation of specific genes). Consecutive eligible patients were offered participation in clinical trials. All patients gave written informed consent and the study and all treatments were conducted in accordance with the guidelines of the Institutional Review Board at Ospedale Niguarda.

The presence of the particular biomarker was investigated according to specific study protocol criteria or retrieved by medical history, where applicable. Further molecular characterization of Kirsten rat sarcoma viral oncogene homolog (KRAS) mutations, a well-known biomarker associated with poor prognosis, was performed in all available samples of patients included in the studies by the Pathology Department of NCC as per standard of care. The registration of patients in the database, pathology assessment, and additional mutation analyses if needed were performed at NCC.

\subsection{Treatment and Evaluation}

Treatment was carried out according to the specific requisites of treatment protocols and was continued until disease progression, unacceptable toxicity, or withdrawal of consent. Assessments, including history, physical examination, and laboratory evaluations, were performed as specified in each protocol, typically before the initiation of therapy, and longitudinally during the treatment cycles. Efficacy was assessed from computed tomography scans and/or magnetic resonance imaging at baseline before treatment initiation and then every 6-8 weeks depending on the study protocol. All radiographs were evaluated by the Department of Radiology at NCC and responses were categorized per RECIST (Response Evaluation Criteria In Solid Tumors) 1.1 criteria and were 
Table 1 Distribution of patients in clinical trials with actionable molecular alterations treated with matched targeted agents included in the pooled analysis

\begin{tabular}{|c|c|c|c|}
\hline Biomarker & Study drug(s) & EudraCT $\mathrm{N}^{\circ}$ & Patients $(n)$ \\
\hline$M G M T$ promoter hypermethylation & Temozolomide [9] & 2012-003338-17 & 27 \\
\hline HER2 amplification & Trastuzumab + lapatinib [10] & $2012-002128-33$ & 23 \\
\hline$M G M T$ promoter hypermethylation & Dacarbazine [11] & 2011-002080-21 & 12 \\
\hline$B R A F$ mutation & MEK162 + LGX818 [NCT01543698] & $2011-005875-17$ & 9 \\
\hline$B R A F$ mutation & MEK162 + panitumumab [NCT01927341] & 2013-001986-18 & 7 \\
\hline$N T R K$ or $A L K$ gene fusions & Entrectinib [12] & $2012-000148-88$ & 2 \\
\hline
\end{tabular}

reported as best response [13]. Progression-free survival of the treatment $\left(\mathrm{PFS}_{\mathrm{n}}\right)$ was recorded for each individual patient as in the case report form of the respective trial, whereas progression-free survival achieved with the previous treatment line received by the patient $\left(\mathrm{PFS}_{\mathrm{n}-1}\right)$ was calculated based on available clinical documentation.

\subsection{Statistical Analysis}

Fisher's exact test was used to assess the association among categorical variables and the presence of the biomarker used for treatment selection. Progression-free survival (PFS) was defined as the time interval from the start of therapy to the first observation of disease progression or death, whichever occurred first. All tests were 2-sided, and $p<0.05$ was considered statistically significant. Multivariate analysis was performed by Cox logistic regression; the model as a whole was evaluated by likelihood ratio test, whereas the significance of the single independent variables was evaluated by means of the Wald test. Results of the multivariate analysis should be interpreted with caution due to the small sample size and sampling bias of our study.

PFS and overall survival (OS) for all patients were computed using the Kaplan-Meier method and differences in PFS and OS were calculated with log-rank test where applicable. The growth modulation index (GMI) has been applied as previously described [14]. We consider a GMI $>1.33$, i.e., an increase in the $\mathrm{PFS}_{\mathrm{n}} / \mathrm{PFS}_{\mathrm{n}-1}$ ratio of $30 \%$, as clinically meaningful. All statistical analyses were carried out using $\mathrm{R}$ software [15] and graphics were generated with ggplot2 [16].

\section{Results}

From June 2011 to May 2016, 2044 patients with mCRC were referred to $\mathrm{NCC}$ for molecular screening for the inclusion into phase I/II trials encompassing the targeting of actionable molecular alterations or molecular contexts of susceptibility. In total, 80 patients $(3.9 \%)$ were enrolled into six ad hoc studies: one phase I/II and five phase II trials.

The median age was 60 years (range 36-86) and the median number of previous treatment lines was five (range 2-8). The six studies included therapies based on $M G M T$ promoter hypermethylation (48.7\%), HER2 amplification $(28.8 \%), B R A F^{V 600 E}$ mutation $(20 \%)$, and gene fusions involving $A L K$ or TRKA (2.5\%) (Table 1 and Fig. 1). Among the 78 of 80 patients evaluable for $K R A S$ mutations, any KRAS (exon 2) mutation was found in $30(38.5 \%)$ of patients.

According to RECIST 1.1 criteria, one patient (1\%) had complete response (CR), 13 patients $(16.5 \%)$ had partial response (PR), and 28 (35\%) had stable disease (SD), accounting for a $52.5 \%$ disease control rate $(\mathrm{DCR}=$ $\mathrm{CR}+\mathrm{PR}+\mathrm{SD})$. The DCR was higher in patients with $K R A S$ wild-type tumors (66 vs. $38 \%, p=0.02$ ). The median PFS was 2.8 months (range 2.63-3.83) (Fig. 2a), and $24 \%$ of patients displayed a PFS $>5$ months. A multivariate analysis of clinicopathological factors including age, gender, performance status, carcinoembryonic antigen (CEA) levels, and KRAS status showed that KRAS status and age were significantly associated with PFS ( $p=0.011$ and 0.047 , respectively), whereas CEA and age were significantly associated with OS ( $p=0.0063$ and 0.0279 , respectively). Patients with KRAS wild-type tumors had longer PFS than those with mutated tumors (3.80 [95\% CI 2.80-5.03] vs. 2.13 months [95\% CI 1.77-2.87], respectively) ( $p=0.001$ ) (Fig. 2b). Median OS was 7.83 months (range 7.17-9.33) (Fig. 3a), and patients with $K R A S$ wild-type tumors had longer OS than those with mutated tumors (7.83 [95\% CI 7.33-10.80] vs. 7.18 months [95\% CI 5.63-9.33], respectively, $p=0.06$ ) (Fig. 3b). The median GMI was 0.85 (range $0-15.61$ ) and $32.5 \%$ of patients had a GMI $>1.33$ (Fig. 4). The Spearman Rho between $\mathrm{PFS}_{\mathrm{n}}$ and $\mathrm{PFS}_{\mathrm{n}-1}$ was 0.10 $(p=0.372)$. 
Fig. 1 RECIST (Response Evaluation Criteria In Solid Tumors) objective response rates according to molecular targets in the pooled patient population. $P R$ partial response, $S D$ stable disease, $P D$ progressive disease, $N A$ not assessed

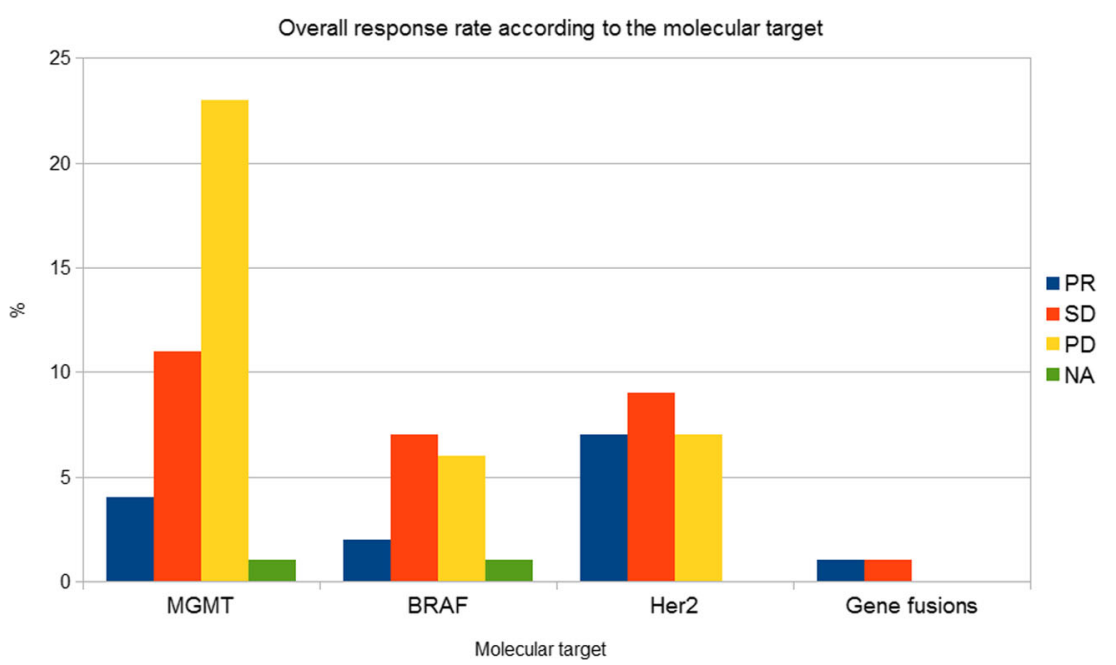

\section{Discussion}

In this single-institution retrospective analysis we found that $4.0 \%$ of mCRC patients refractory to standard therapies were eligible for enrollment into phase I/II clinical trials based on molecular target selection. These patients, who had experienced a median number of five lines of previous treatments, achieved a median PFS of 2.8 months (with approximately one-quarter of patients displaying a PFS $>5$ months) and a median OS of 7.8 months. These data should be evaluated in the context of advanced mCRC, where the median PFS with newer agents has been less than 2 months [20], and rechallenge with standard chemotherapy $[18,19]$ or further chemotherapy [17] showed only poor efficacy and was burdened by adverse effects [21]. With regards to objective response rates (ORRs), 14 patients (17.5\%) had PR and 28 (35\%) SD, accounting for a 52.5\% DCR. These data compare favorably with those achieved with licensed third-line therapies such as the multikinase inhibitor regorafenib $(\mathrm{ORR}=1-4 \%)$ and the trifluridine-tipiracil combination TAS-102 (ORR $=2 \%)$, both of which are administered without the selection of a molecular target, or salvage chemotherapy with mitomycin-C or oxaliplatin rechallenge [17, 22, 23].

Following the seminal work by von Hoff et al. [14], several reports investigating the use of molecularly targeted agents on the basis of the identification of molecular alterations provided conflicting results [43, 44]. Provocative findings from Janku et al. highlighted that in phase I studies the lack of matching between molecular alterations and targeted treatment provides only limited clinical benefit in CRC and other solid tumors [24]. Recently, the same authors reported findings of a prospective, single-center 'navigation' study conducted in 500 patients with several different refractory cancers who underwent comprehensive genomic profiling by sequencing of 236 genes, offering proof-of-concept for the utility of this approach in assigning therapy to patients with refractory malignancies, especially in those patients with multiple genomic aberrations for whom combination therapies could be implemented [25]. However, patients with CRC were only a small proportion of this cohort and therefore definitive conclusions could not be drawn for this histology. In mCRC, Dienstmann et al. reported a series of 68 patients treated with targeted agents in phase I trials based on molecular profiling, concluding that this approach did not confer a significant clinical benefit on the basis of a comparison between the median time to treatment failure $n\left(\mathrm{TTF}_{\mathrm{n}}\right)$ of 7.9 weeks and $\mathrm{TTF}_{\mathrm{n}-1}$ of 16.3 weeks [26]. Similarly, in our present analysis of 80 patients, we observed that $\mathrm{PFS}_{\mathrm{n}}$ was inferior to $\mathrm{PFS}_{\mathrm{n}-1}$ (12.0 vs. 15.6 weeks). However, there are substantial differences between these two cohorts. Our series included only $2.5 \%$ patients treated within a phase I study versus $100 \%$ of the patients included in the study by Dienstmann et al. [26], and there are differences in the molecular alterations on which the treatments are based (KRAS/BRAF/PIK3CA mutations, PTEN and MET expression vs. HER2, BRAF, MGMT, and gene fusions, respectively). Assessment of clinical benefit by means of the ratio between PFS with the actual targeted therapy and that achieved with the previous line of treatment, referred to as the GMI, has also been proposed [14]. When we applied this index to our cohort, we found that at least onethird of patients benefited from the enrollment in clinical trials with targeted agents matched to selected actionable molecular alterations, making our results meaningful.

Among the molecular abnormalities selected in our cohort, we included gene fusions involving the $A L K$ and NTRK genes. These alterations have only recently been recognized as targets occurring at low prevalence in CRC [27]. In particular, in 2014 we reported the characterization of the TPM3-NTRK1 gene rearrangement as a recurring, although rare, event in CRC. The concomitant discovery of entrectinib (NMS-P626; RXDX-101) as a novel highly potent and selective pan-Trk inhibitor by the group of Ardini et al. [28] enhanced the interest in this target, with further development across different 
Fig. 2 Progression-free survival $\mathbf{a}$ for all patients; and $\mathbf{b}$ according to $K R A S$ status

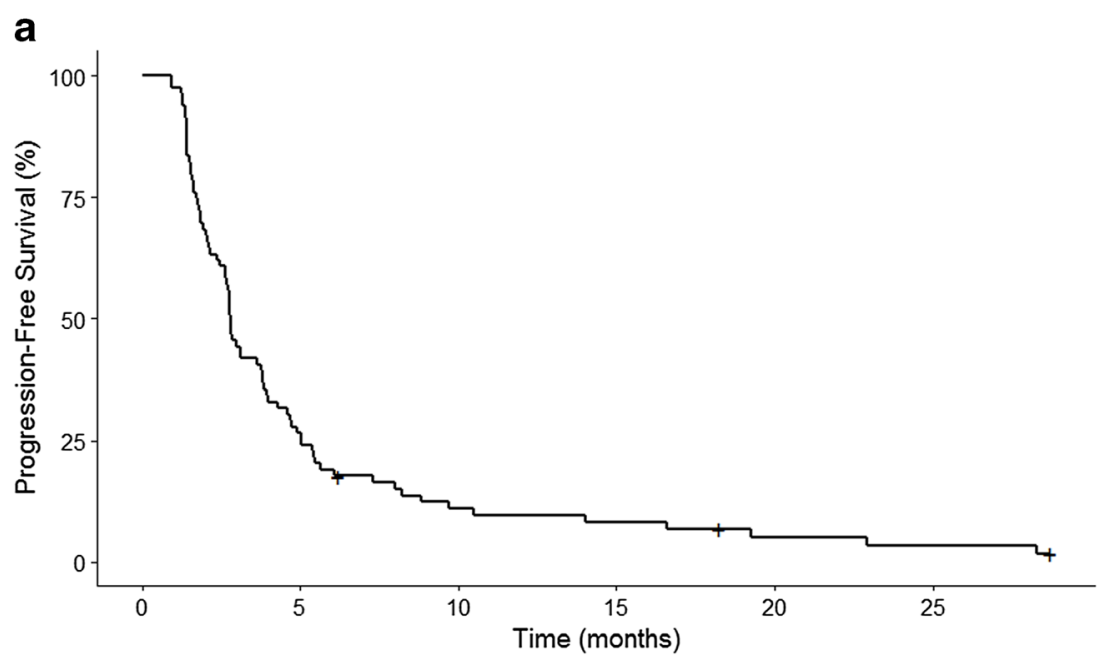

Number at risk

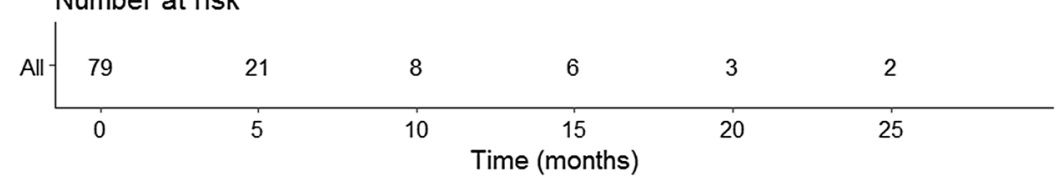

b

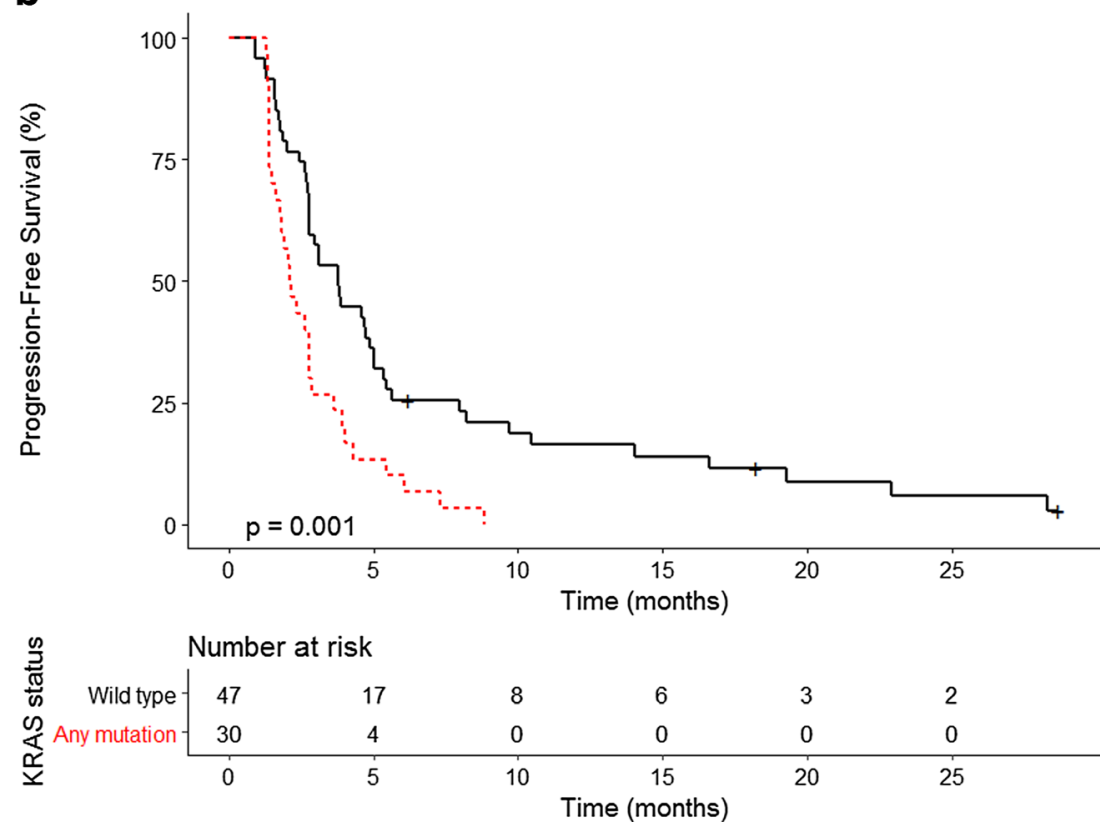

histologies and currently ongoing phase II studies, including in CRC $[12,27]$. Interestingly enough, the two gene fusions involving NTRK and $A L K$ described in the present cohort were previously unreported $[29,30]$, highlighting the challenge of a comprehensive molecular pathology that should not merely include gene fusions together with mutations and amplifications but also elucidate the exact fusion partner and oncogenic potential for treatment with specific inhibitors.

During the last decade, the assessment of KRAS and neuroblastoma RAS viral oncogene homolog (NRAS) gained increasing importance for an optimal management of $\mathrm{mCRC}$
[31]. Mutations in $K R A S$ codons 12 or 13 (in exon 2) affect approximately $40 \%$ of patients with metastatic CRC [32]. About an additional $5 \%$ of CRC patients display mutations in $K R A S$ exons 3 or 4, usually at codon 61 or 146, and another $5 \%$ of CRC patients have mutations in NRAS in exons 2, 3, or 4. Extended $R A S$ status (KRAS and NRAS exons 2, 3, and 4 mutations) is the validated biomarker of response to antiEGFR antibodies [31]. RAS mutant mCRC exhibit a significantly higher cumulative incidence of lung, bone, and brain metastasis [33-35]. The prognostic role of $R A S$ mutations is controversial, but increasing evidence shows a negative effect 
Fig. 3 Overall survival a for all patients; and $\mathbf{b}$ according to $K R A S$ status a
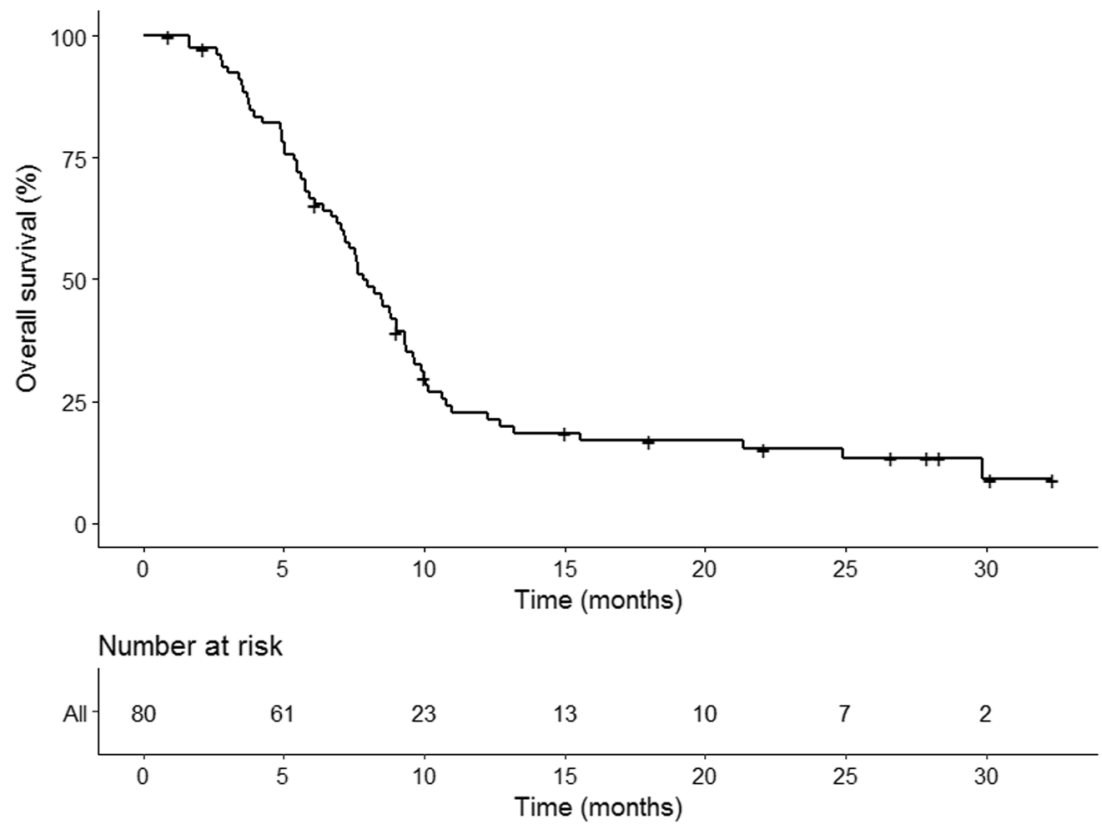

b

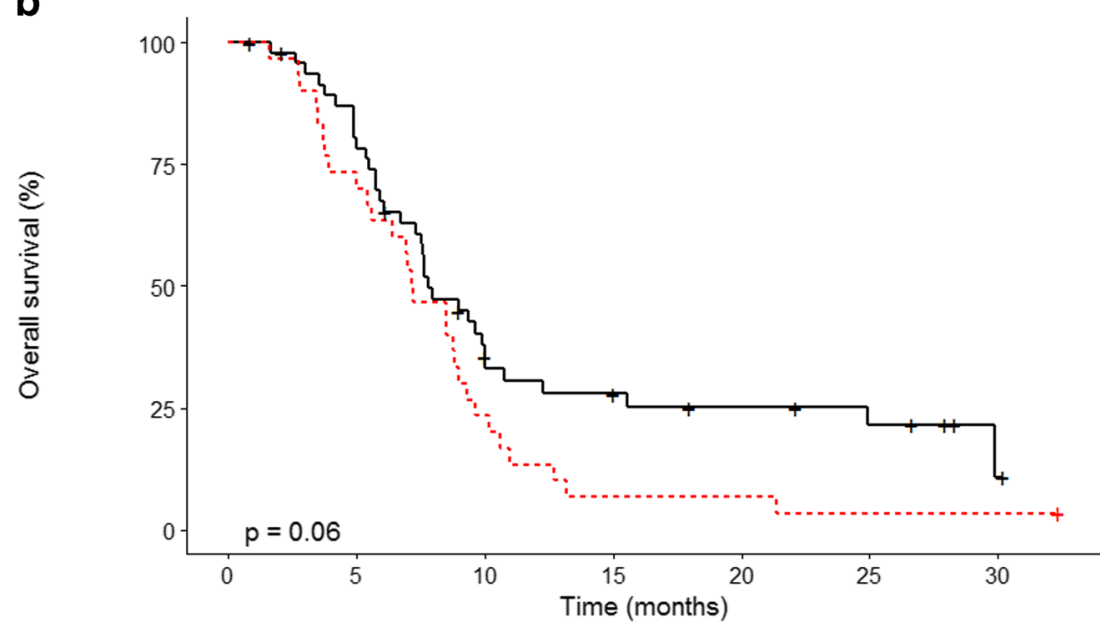

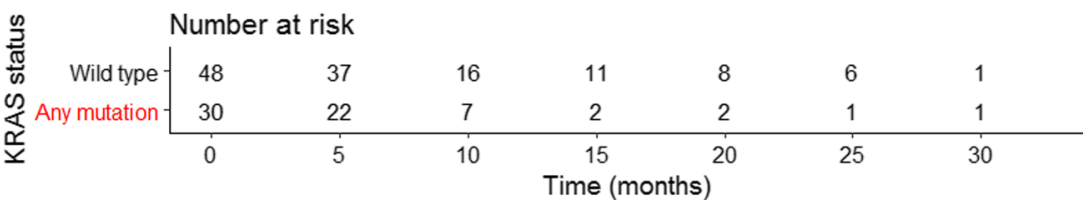

in the adjuvant and metastatic setting [36-38]. In our cohort, $K R A S$ mutations showed an overall negative prognostic value, since patients harboring KRAS-mutated tumors had shorter PFS and OS. Also, DCR was more frequent in patients with $K R A S$ wild-type tumors. However, it should be taken into account that half of the selected phase I/II trials were designed for patients with KRAS wild-type tumors (Cmek2110 and Cmek2116 for BRAF-mutated tumors, HER2 Amplification for Colo-rectaL Cancer Enhanced Stratification-HERACLES for HER2 amplified tumors) and most of the KRAS-mutated patients were treated in the two studies (DETECT and TEMECT with dacarbazine and temozolomide, respectively) that were not based on directly targeting an oncogenic driver but rather exploiting a molecular context of susceptibility.

Limitations of our study include the small sample size and potential sampling bias, the notion that the use of GMI as an index of clinical benefit in CRC has been criticized as there is a lack of correlation between $\mathrm{PFS}_{\mathrm{n}}$ and $\mathrm{PFS}_{\mathrm{n}-1}$ that is the 


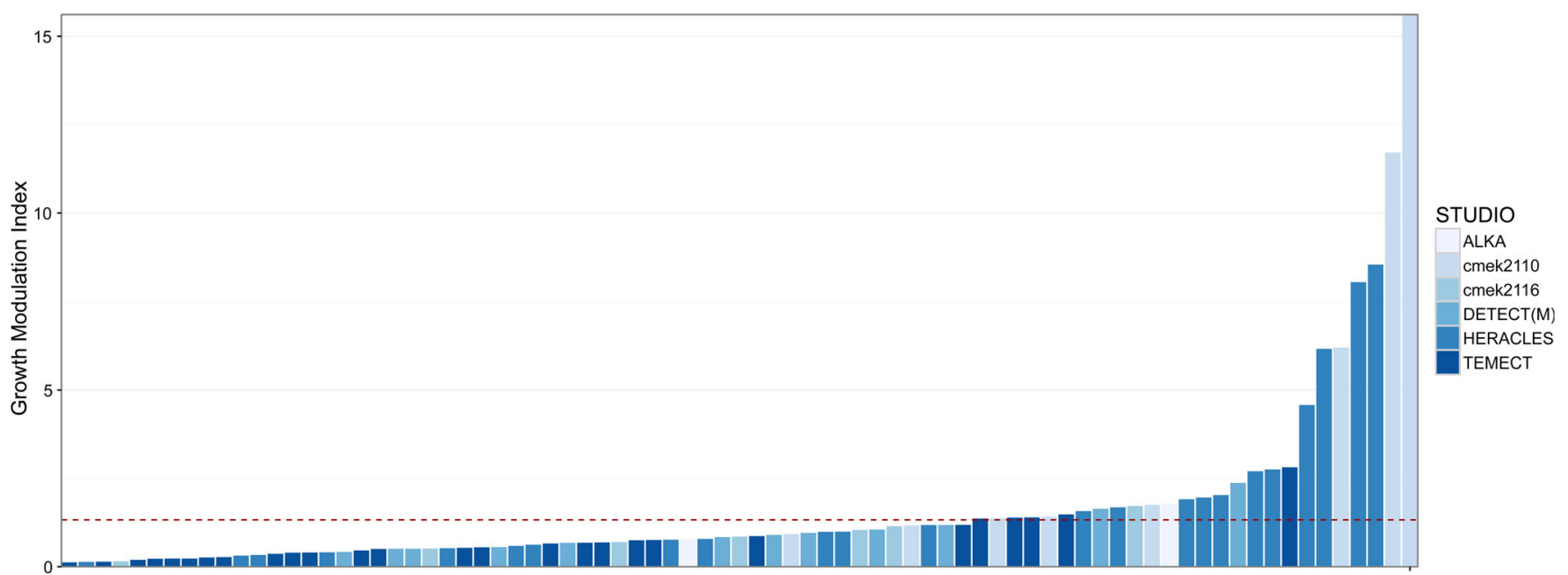

Fig. 4 Growth modulation index for each patient included in the pooled analysis and corresponding phase I/II clinical trials. $M *$ subset of patients within the study with hypermethylation of $M G M T$

statistical assumption for applying this method [39, 40], and that KRAS-limited instead of extended $R A S$ analysis was performed in our cohort.

In conclusion, our single-institution study indicates that in a heavily pretreated mCRC population about $4 \%$ of tumors display a potential actionable molecular context suitable for phase I/II trials with matched therapeutics. Comprehensive molecular selection is challenging, encompassing inclusion of actionable alterations occurring at low frequency in CRC such as HER2 amplification [10] and rare gene fusions involving the $A L K$ and NTRK genes [41], but enhances therapeutic options to be exploited in the advanced setting. This knowledge, coupled with recent advancements in the understanding of acquired resistance mechanisms to targeted agents through liquid biopsy [8, 42] is expanding opportunities of precision oncology therapies for CRC.

Acknowledgments The Authors are profoundly grateful to Professor Alberto Bardelli who provided knowledge and cultural motivation to the Precision Oncology Program at Niguarda Cancer Center.

Author Contributions AS-B provided patients' care, collected patients' samples, supervised data collection and analysis, and was the major contributor in writing the manuscript. AA provided patients' care, participated in data collection, statistical analysis, and writing the manuscript. EB provided patients' care and participated in data collection and writing the manuscript. SSt performed data collection and participated in collection of patients' samples. LG, GC, IS, KB, CF, RR, TC, SDB, MS, VG, LP, GC-S, FR, GB and ET provided patients' care. FP, SG, AC and AG participated in data and patients' samples collection. GM supervised data and patients' samples collection. SV EV, CL and MT performed diagnostic pathology and molecular analysis on tumor tissue specimens as per clinical studies. AV supervised local radiological assessment of patients as per clinical studies. SSi supervised patients' care and participated in data analysis and writing of the manuscript. All authors read and approved the final manuscript.

\section{Compliance with Ethical Standards}

Funding The study was partly supported by the grant "Terapia Molecolare dei Tumori” by Fondazione Oncologia Niguarda, by Associazione Italiana per la Ricerca sul Cancro (AIRC) Special program $5 \times 1000$, and by the European Union Horizon 2020 research and innovation programme 2014-2020 under grant agreement number 635342 (MoTriColor project).

Conflicts of Interest The authors declare no conflicts of interest.

Open Access This article is distributed under the terms of the Creative Commons Attribution-NonCommercial 4.0 International License (http:// creativecommons.org/licenses/by-nc/4.0/), which permits any noncommercial use, distribution, and reproduction in any medium, provided you give appropriate credit to the original author(s) and the source, provide a link to the Creative Commons license, and indicate if changes were made.

\section{References}

1. Miller KD, Siegel RL, Lin CC, Mariotto AB, Kramer JL, Rowland $\mathrm{JH}$, et al. Cancer treatment and survivorship statistics, 2016. CA Cancer J Clin. 2016;66:271-89.

2. Abdalla EK, Vauthey J-N, Ellis LM, Ellis V, Pollock R, Broglio $\mathrm{KR}$, et al. Recurrence and outcomes following hepatic resection, radiofrequency ablation, and combined resection/ablation for colorectal liver metastases. Ann Surg. 2004;239:818-25.

3. Adam R, Delvart V, Pascal G, Valeanu A, Castaing D, Azoulay D, et al. Rescue surgery for unresectable colorectal liver metastases downstaged by chemotherapy: a model to predict long-term survival. Ann Surg. 2004;240:644-57.

4. Siegel R, DeSantis C, Jemal A. Colorectal cancer statistics, 2014. CA Cancer J Clin. 2014;64:104-17.

5. Longley DB, Johnston PG. Molecular mechanisms of drug resistance. J Pathol. 2005;205:275-92.

6. Cunningham D, Humblet Y, Siena S, Khayat D, Bleiberg H, Santoro A, et al. Cetuximab monotherapy and cetuximab plus irinotecan in irinotecan-refractory metastatic colorectal cancer. $\mathrm{N}$ Engl J Med. 2004;351:337-45. 
7 Siravegna G, Mussolin B, Buscarino M, Corti G, Cassingena A, Crisafulli $\mathrm{G}$, et al. Clonal evolution and resistance to EGFR blockade in the blood of colorectal cancer patients. Nat Med. 2015;21: 795-801.

8 Sartore-Bianchi A, Loupakis F, Argilés G, Prager GW. Challenging chemoresistant metastatic colorectal cancer: therapeutic strategies from the clinic and from the laboratory. Ann Oncol. 2016;27:1456-66.

9 Amatu A, Barault L, Moutinho C, Cassingena A, Bencardino K, Ghezzi $\mathrm{S}$, et al. Tumor MGMT promoter hypermethylation changes over time limit temozolomide efficacy in a phase II trial for metastatic colorectal cancer. Ann Oncol. 2016;27:1062-7.

10 Sartore-Bianchi A, Trusolino L, Martino C, Bencardino K, Lonardi $\mathrm{S}$, Bergamo F, et al. Dual-targeted therapy with trastuzumab and lapatinib in treatment-refractory, KRAS codon 12/13 wild-type, HER2-positive metastatic colorectal cancer (HERACLES): a proof-of-concept, multicentre, open-label, phase 2 trial. Lancet Oncol. 2016;17:738-46.

11 Amatu A, Sartore-Bianchi A, Moutinho C, Belotti A, Bencardino K, Chirico G, et al. Promoter CpG island hypermethylation of the DNA repair enzyme MGMT predicts clinical response to dacarbazine in a phase II study for metastatic colorectal cancer. Clin Cancer Res. 2013;19:2265-72.

12 Drilon A, Siena S, Ou SI, Patel M, Ahn MJ, Lee J, et al. Safety and Antitumor Activity of the Multitargeted Pan-TRK, ROS1, and ALK Inhibitor Entrectinib: Combined Results from Two Phase I Trials (ALKA-372-001 and STARTRK-1). Cancer Discov. 2017;4:400-9.

13 Eisenhauer EA, Therasse P, Bogaerts J, Schwartz LH, Sargent D, Ford R, et al. New response evaluation criteria in solid tumours: revised RECIST guideline (version 1.1). Eur J Cancer 1990. 2009;45:228-47.

14 Von Hoff DD, Stephenson JJ, Rosen P, Loesch DM, Borad MJ, Anthony S, et al. Pilot study using molecular profiling of patients' tumors to find potential targets and select treatments for their refractory cancers. J Clin Oncol. 2010;28:4877-83.

15 R Development Core Team. R: a language and environment for statistical computing [Internet]. Vienna: R Foundation for Statistical Computing; 2008. http://www.R-project.org

16 Wickham H. ggplot2: elegant graphics for data analysis [Internet]. New York: Springer-Verlag; 2009. http://ggplot2.org

17 Petrelli F, Ghidini A, Inno A, Barni S. Mitomycin-C+ fluoropyrimidines in heavily pretreated metastatic colorectal cancer: a systematic review and evidence synthesis. Anti-Cancer Drugs. 2016;27:488-95.

18 Townsend AR, Bishnoi S, Broadbridge V, Beeke C, Karapetis CS, Jain $\mathrm{K}$, et al. Rechallenge with oxaliplatin and fluoropyrimidine for metastatic colorectal carcinoma after prior therapy. Am J Clin Oncol. 2013;36:49-52.

19 Santini D, Vincenzi B, La Cesa A, Caricato M, Schiavon G, Spalletta B, et al. Continuous infusion of oxaliplatin plus chronomodulated capecitabine in 5-fluorouracil- and irinotecan-resistant advanced colorectal cancer patients. Oncology. 2005;69:27-34.

20 Nielsen DL, Palshof JA, Larsen FO, Jensen BV, Pfeiffer P. A systematic review of salvage therapy to patients with metastatic colorectal cancer previously treated with fluorouracil, oxaliplatin and irinotecan $+/-$ targeted therapy. Cancer Treat Rev. 2014;40:701-15.

21 Bencardino K, Mauri G, Amatu A, Tosi F, Bonazzina E, Palmeri L, et al. Oxaliplatin immune-induced syndrome occurs with cumulative administration and Rechallenge: single institution series and systematic review study. Clin Colorectal Cancer. 2016;15:213-21.

22 Grothey A, Van Cutsem E, Sobrero A, Siena S, Falcone A, Ychou $\mathrm{M}$, et al. Regorafenib monotherapy for previously treated metastatic colorectal cancer (CORRECT): an international, multicentre, randomised, placebo-controlled, phase 3 trial. Lancet. 2013;381: $303-12$.
23 Mayer RJ, Van Cutsem E, Falcone A, Yoshino T, Garcia-Carbonero R, Mizunuma N, et al. Randomized trial of TAS-102 for refractory metastatic colorectal cancer. N Engl J Med. 2015;372:1909-19.

24 Janku F, Lee JJ, Tsimberidou AM, Hong DS, Naing A, Falchook GS, et al. PIK3CA mutations frequently coexist with RAS and BRAF mutations in patients with advanced cancers. PLoS One. 2011;6:e22769.

25 Wheler JJ, Janku F, Naing A, Li Y, Stephen B, Zinner R, et al. Cancer therapy directed by comprehensive genomic profiling: a single center study. Cancer Res. 2016;76:3690-701.

26 Dienstmann R, Serpico D, Rodon J, Saura C, Macarulla T, Elez E, et al. Molecular profiling of patients with colorectal cancer and matched targeted therapy in phase I clinical trials. Mol Cancer Ther. 2012;11:2062-71.

27 Amatu A, Sartore-Bianchi A, Siena S. NTRK gene fusions as novel targets of cancer therapy across multiple tumour types. ESMO Open. 2016;1:e000023.

28 Ardini E, Menichincheri M, Banfi P, Bosotti R, De Ponti C, Pulci R, et al. Entrectinib, a pan-TRK, ROS1, and ALK inhibitor with activity in multiple molecularly defined cancer indications. Mol Cancer Ther. 2016;15:628-39.

29 Amatu A, Somaschini A, Cerea G, Bosotti R, Valtorta E, Buonandi $\mathrm{P}$, et al. Novel CAD-ALK gene rearrangement is drugable by entrectinib in colorectal cancer. Br J Cancer. 2015;113:1730-4.

30 Sartore-Bianchi A, Ardini E, Bosotti R, Amatu A, Valtorta E, Somaschini A, et al. Sensitivity to entrectinib associated with a novel LMNA-NTRK1 gene fusion in metastatic colorectal cancer. J Natl Cancer Inst 2016;108.

31 Douillard J-Y, Oliner KS, Siena S, Tabernero J, Burkes R, Barugel $\mathrm{M}$, et al. Panitumumab-FOLFOX4 treatment and RAS mutations in colorectal cancer. N Engl J Med. 2013;369:1023-34.

32 Moroni M, Veronese S, Benvenuti S, Marrapese G, Sartore-Bianchi A, Di Nicolantonio F, et al. Gene copy number for epidermal growth factor receptor (EGFR) and clinical response to antiEGFR treatment in colorectal cancer: a cohort study. Lancet Oncol. 2005;6:279-86.

33 Cejas P, López-Gómez M, Aguayo C, Madero R, de Castro CJ, Belda-Iniesta $\mathrm{C}$, et al. KRAS mutations in primary colorectal cancer tumors and related metastases: a potential role in prediction of lung metastasis. PLoS One. 2009;4:e8199.

34 Andreyev HJN, Norman AR, Cunningham D, Oates J, Dix BR, Iacopetta BJ, et al. Kirsten ras mutations in patients with colorectal cancer: the "RASCAL II" study. Br J Cancer. 2001;85:692-6.

35 Deng Y, Wang L, Tan S, Kim GP, Dou R, Chen D, et al. KRAS as a predictor of poor prognosis and benefit from postoperative FOLFOX chemotherapy in patients with stage II and III colorectal cancer. Mol Oncol. 2015;9:1341-7.

36 Kadowaki S, Kakuta M, Takahashi S, Takahashi A, Arai Y, Nishimura Y, et al. Prognostic value of KRAS and BRAF mutations in curatively resected colorectal cancer. World J Gastroenterol. 2015;21:1275-83.

37 Vincenzi B, Cremolini C, Sartore-Bianchi A, Russo A, Mannavola F, Perrone G, et al. Prognostic significance of K-Ras mutation rate in metastatic colorectal cancer patients. Oncotarget. 2015;6:31604-12.

38 Tosi F, Magni E, Amatu A, Mauri G, Bencardino K, Truini M, et al. Effect of KRAS and BRAF mutations on survival of metastatic colorectal cancer after liver resection: a systematic review and meta-analysis. Clin Colorectal Cancer. Epub 2017 Jan 25. doi: 10.1016/ j.clcc.2017.01.004.

39 Mick R, Crowley JJ, Carroll RJ. Phase II clinical trial design for noncytotoxic anticancer agents for which time to disease progression is the primary endpoint. Control Clin Trials. 2000;21:343-59.

40 Buyse M, Quinaux E, Hendlisz A, Golfinopoulos V, Tournigand C, Mick R. Progression-free survival ratio as end point for phase II trials in advanced solid tumors. J Clin Oncol. 2011;29:e451-2. 
41 Overman MJ, Morris V, Kee B, Fogelman D, Xiao L, Eng C, et al. Utility of a molecular prescreening program in advanced colorectal cancer for enrollment on biomarker-selected clinical trials. Ann Oncol. 2016;27:1068-74.

42 Russo M, Siravegna G, Blaszkowsky LS, Corti G, Crisafulli G, Ahronian LG, et al. Tumor heterogeneity and lesion-specific response to targeted therapy in colorectal cancer. Cancer Discov. 2016;6:147-53.

43 Le Tourneau C, Delord J-P, Gonçalves A, Gavoille C, Dubot C, Isambert $\mathrm{N}$, et al. Molecularly targeted therapy based on tumour molecular profiling versus conventional therapy for advanced cancer (SHIVA): a multicentre, open-label, proof-of-concept, randomised, controlled phase 2 trial. Lancet Oncol. 2015;16: 1324-34.

44 Tsimberidou A-M, Iskander NG, Hong DS, Wheler JJ, Falchook $\mathrm{GS}, \mathrm{Fu} \mathrm{S}$, et al. Personalized medicine in a phase I clinical trials program: the MD Anderson Cancer Center initiative. Clin Cancer Res. 2012;18:6373-83.

45 Price TJ, Peeters M, Kim TW, Li J, Cascinu S, Ruff P, et al. Panitumumab versus cetuximab in patients with chemotherapyrefractory wild-type KRAS exon 2 metastatic colorectal cancer (ASPECCT): a randomised, multicentre, open-label, noninferiority phase 3 study. Lancet Oncol. 2014;15:569-79. 